\title{
Visceral Fat Accumulation Is Related to Impaired Pancreatic Blood Perfusion and Beta-Cell Dysfunction in Obese Women
}

\author{
Radka Szotkowskáa, ban Gojda ${ }^{a, b}$ Andrea Plíhalováa, ${ }^{a}$ Jiří Weichet ${ }^{c}$ \\ Jana Potočkováa, b Jan Havlík ${ }^{e}$ Jan Polák ${ }^{a, d}$ Michal Anděl ${ }^{a, b}$ \\ aDepartment of Internal Medicine 2, University Hospital Královské Vinohrady and Third Faculty of Medicine, Charles \\ University, Prague, Czech Republic; ${ }^{b}$ Centre for Research on Nutrition, Metabolism and Diabetes of Third Faculty \\ of Medicine, Charles University, Prague, Czech Republic; 'Department of Radiodiagnostics, University Hospital \\ Královské Vinohrady and Third Faculty of Medicine, Charles University, Prague, Czech Republic; ${ }^{d}$ Department of

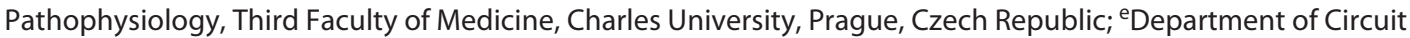 \\ Theory, Faculty of Electrical Engineering, Czech Technical University in Prague, Prague, Czech Republic
}

\section{Keywords}

Obesity · Pancreas · Blood perfusion - Type 2 diabetes mellitus

\begin{abstract}
Aims/Hypothesis: Beta-cell failure plays a fundamental role in type 2 diabetes mellitus (T2DM) development. It has been shown that the beta-cells are among the most sensitive to hypoxia. We aimed to analyze whether decrease in pancreatic perfusion relates to $1 /$ decline in beta-cell function and 2/visceral fat accumulation in patients with T2DM. Methods: Fifteen women with T2DM on metformin therapy alone and fifteen women of comparable age and BMI without prediabetes/diabetes were cross-sectionally examined: clinical and anthropometric examination, fast sampled intravenous glucose tolerance test (FSIVGTT), dynamic contrast-enhanced magnetic resonance imaging to assess pancreatic perfusion (area under the curve of postcontrast saturation, $\mathrm{AUC}_{\mathrm{TSIC}}$ ), and visceral adiposity (VAT, calculated from transverse sections at the level L2-L5 vertebrae). Results: Pancreatic blood perfusion ( $\left.A \cup C_{T S I C}\right)$ did not differ between groups $(p=0.273)$,
\end{abstract}

but it negatively correlated with BMI $(r=-0.434, p=0.017)$, WHR $(r=-0.411, p=0.024)$, and VAT $(r=-0.436, p=0.016)$ in both groups. Moreover, $\mathrm{AUC}_{\mathrm{TSIC}}$ in the head of the pancreas negatively correlated with the level of fasting glycemia $(r=-0.401, p=0.028)$ and HOMA-IR $(r=-0.376, p=0.041)$. Discussion/Conclusion: We showed that decreased pancreatic perfusion did not relate to beta-cell dysfunction in early stages of T2DM development, but it was related to VAT, insulin resistance, and higher fasting glycemia. Furthermore, lower pancreatic perfusion was related to VAT, insulin resistance, and higher fasting glycemia.

(C) 2021 The Author(s).

Published by S. Karger AG, Basel

\section{Introduction}

The rising incidence of obesity in recent decades makes it one of the leading health problems of modern medicine, as the disease is associated with many serious consequences such as cardiovascular and oncological diseases or type 2 diabetes mellitus (T2DM). T2DM occurs when the established insulin resistance of the tissues can no lon- karger@karger.com www.karger.com/anm

Karger $\stackrel{\text { ' }}{5}$

BOPEN ACCESS
(C) 2021 The Author(s)

Published by S. Karger AG, Basel

This article is licensed under the Creative Commons Attribution 4.0 International License (CC BY) (http://www.karger.com/Services/ OpenAccessLicense). Usage, derivative works and distribution are permitted provided that proper credit is given to the author and the original publisher.
Correspondence to:

Radka Szotkowská, radka.szotkowska@gmail.com 
ger be compensated by insulin hypersecretion. Thus, beta-cell failure develops. Beta-cell function and viability are negatively affected by hyperglycemia, increased concentrations of trans and saturated fatty acids, oxygen radicals and some drugs [1], and hypoxia, which we focused on here. Only $2 \%$ of the volume of the pancreas is made up of the islets of Langerhans, but they consume almost $20 \%$ of the arterial blood supply [2], so that even a small disorder in the blood supply to the pancreas can be reflected in their function.

Both disorder of insulin secretion and increase in insulin resistance were observed in a mouse model exposed to intermittent hypoxia, and after normalization of oxygen supply, these disorders were partially corrected [3]. Such hypoxia can certainly occur when the blood supply to the pancreatic islets is disrupted. In animal models of T2DM, initial islet hyperperfusion was observed in the prediabetic and early stages of the disease followed by late hypoperfusion and these changes could be prevented by food restrictions [4]. And it has been shown that pancreatic tissue hypoxia leads to increased levels of apoptosis in beta-cells, but is not affected by islet alpha-cells [5]. However, the extent to which changes in blood perfusion may affect the pathogenesis of pancreatic disease is unknown. The aim of our project was to determine pancreatic blood perfusion in obese nondiabetic and T2DM women using dynamic contrast-enhanced magnetic resonance imaging (DCE-MRI) [6-8] and to relate the obtained data to beta-cell functional parameters and insulin sensitivity determined by the fast sampled intravenous glucose tolerance test (FSIVGTT) [9] to anthropometric parameters and to the amount of VAT calculated from MR examination.

\section{Materials and Methods}

\section{Subjects}

Fifteen overweight/obese women (BMI above $25 \mathrm{~kg} / \mathrm{m}^{2}$ ) diagnosed with T2DM on metformin therapy alone and 15 women of comparable age $(62.7 \pm 1.4$ vs. $62.9 \pm 1.6, p=0.901)$, BMI $(35.3 \pm$ 1.0 vs. $33.2 \pm 1.4, p=0.23$ ), and physical activity (Baecke score 7.27 \pm 0.17 vs. $7.85 \pm 0.49, p=0.271$ ) without impaired glucose metabolism were enrolled. All women were postmenopausal. The average duration of diabetes in the first group was 5.5 years (range 1-17 years, median 5 years). Patients with T2DM did not take any drugs interfering with beta-cell function (i.e., other antidiabetic drugs, etc.). The exclusion criteria were kidney, liver, or heart disease; medical history of any atherosclerotic/cardiovascular disease; unstable thyroid disease; and, due to the use of a magnetic resonance device, also body weight over $130 \mathrm{~kg}$ or implanted ferromagnetic materials per MRI examination. All women participated voluntarily and signed an informed consent prior to enrollment in the study. The research protocols were approved by the Ethical Committee of the University Hospital Kralovske Vinohrady (Approval Number EK-VP/30/0/2016) in accordance with the Declaration of Helsinki, and the study was conducted in accordance with ICHGCP.

\section{Methods}

Clinical Examination and FSIVGTT

Blood collection for routine laboratory analysis (blood count, HDL, LDL, total cholesterol, TAG, fasting blood glucose, fasting plasma insulin, HbA1c, TSH, ALT, AST, GMT, ALP, urea, creatinine) was performed after $12 \mathrm{~h}$ of overnight fasting. Biochemical testing of all blood samples was performed by standard methods at the Institute of Laboratory Diagnostics, University Hospital Kralovske Vinohrady. HOMA-IR was calculated from the plasma insulin and plasma glucose according to the standard equation [10]. This was followed by detailed anthropometric measurements, bioelectrical impedance examination (NutriGuard M2000; Data-Input, GmbH, Germany), and pulse wave velocity measurements (VaSera VS-1500N; Fukuda Denshi). All subjects also completed the Baecke physical activity questionnaire [11].

Then, FSIVGTT was performed in each subject according to a standard protocol $[9,12]$. After being placed in bed, cannulas were inserted into the peripheral vein of both upper limbs of the patient. One was used for glucose and insulin administration throughout the protocol and the other for blood sampling. At 0 min, a $40 \%$ glucose solution was administered at a dose of $0.3 \mathrm{~g} / \mathrm{kg}$ body weight as a bolus for $60 \mathrm{~s}$. At $20 \mathrm{~min}$, diluted insulin at a dose of $0.03 \mathrm{IU} /$ $\mathrm{kg}$ body weight was administered intravenously over $30 \mathrm{~s}$. Venous blood samples for glucose and insulin analysis were taken at -10 , $-5,-1,2,3,4,5,6,8,10,12,14,16,19,22,24,25,27,30,40,50,60$, $70,80,90,100,120,140,160$, and $180 \mathrm{~min}$. From the obtained data, the following parameters were evaluated using MINMOD software (Richard N. Bergman, Los Angeles, CA, USA): insulin sensitivity (SI) $\left([\mathrm{mU} / \mathrm{L}]^{-1} \times \mathrm{min}^{-1}\right)$, acute insulin response to glucose $(\mathrm{AIRg})\left(\mathrm{mU} \times \mathrm{L}^{-1} \times \mathrm{min}\right)$, and disposition index (DI) which equals $\operatorname{AIRg} \times$ SI.

\section{MR Imaging}

All volunteers underwent abdominal MRI within 14 days after the clinical examination and laboratory tests. The MRI was performed in $1.5 \mathrm{~T}$ scanner (Signa HDxt; GE Healthcare) and consisted of sequences: T1-weighted gradient dual-echo in transversal plane, LAVA-Flex (2-point Dixon) with water and fat reconstructions in transversal plane in breathhold, and dynamic T1-weighted 3D gradient echo key-hole sequence after intravenous injection of gadolinium contrast agent gadobutrol (Gadovist; Bayer Pharma AG) into antecubital vein (dose $0.1 \mathrm{mmol} / \mathrm{kg}$ body weight following $20 \mathrm{~mL}$ saline flush). The dynamic scanning of pancreatic region lasted 2 min during shallow breathing with temporal resolution of $3.2 \mathrm{~s}$. Visceral fat area was manually segmented and measured at levels L2/3, L3/4, and L4/5 using fat reconstructions of LAVA-Flex sequence. Regions of interest were carefully placed by one radiologist with 20 years of experience in cross-sectional abdominal imaging over the head, body, and tail of pancreas as well as abdominal aorta on transverse images of dynamic sequence, signal intensity for each time point in respective areas was recorded, and signal intensity/time curves were constructed. 
Table 1. Characteristics of groups

\begin{tabular}{llll}
\hline & T2DM $(n=15)$ & Obese $(n=15)$ & $p$ value \\
\hline Age, years & $62.68 \pm 1.36$ & $62.94 \pm 1.59$ & 0.90 \\
BMI, kg/m & $35.26 \pm 1.01$ & $33.18 \pm 1.37$ & 0.23 \\
WHR & $0.8951 \pm 0.02$ & $0.8518 \pm 0.01$ & 0.08 \\
Waist, cm & $104.1 \pm 2.43$ & $99.40 \pm 2.91$ & 0.23 \\
Visceral fat, $\mathrm{mm}^{2}$ & $41,536 \pm 34$ & $33,908 \pm 22$ & 0.07 \\
Fat BIA, kg & $32.56 \pm 1.73$ & $32.05 \pm 2.33$ & 0.86 \\
Atherosclerosis score & $1.53 \pm 0.13$ & $1.47 \pm 0.13$ & 0.73 \\
Baecke score & $7.27 \pm 0.17$ & $7.85 \pm 0.49$ & 0.27 \\
SI, mU/L ${ }^{-1} \times$ min $^{-1}$ & $1.762 \pm 0.6$ & $2.330 \pm 0.22$ & 0.38 \\
AlRg, mU $\times \mathrm{L}^{-1} \times \mathrm{min}$ & $172.5 \pm 73.96$ & $513.5 \pm 97.43$ & $0.0094^{* *}$ \\
DI & $262.9 \pm 100.7$ & $1,102 \pm 187.3$ & $0.0002^{* * *}$ \\
HbA1c, mmol/mol & $49.87 \pm 2.04$ & $36.67 \pm 0.77$ & $<0.0001^{* * *}$ \\
Fasting glycemia, $\mathrm{mmol} / \mathrm{L}$ & $7.42 \pm 0.40$ & $5.33 \pm 0.11$ & $<0.0001^{* * *}$ \\
Fasting insulinemia, $\mu \mathrm{IU} / \mathrm{mL}$ & $13.17 \pm 1.27$ & $7.61 \pm 0.53$ & $0.0004^{* * *}$ \\
HOMA-IR & $4.044 \pm 0.403$ & $1.886 \pm 0.14$ & $<0.0001^{* * *}$ \\
\hline
\end{tabular}

Data are means \pm SEM with significance tested using Student's $t$ test. ${ }^{*} p<0.05,{ }^{* *} p<$ $0.01,{ }^{* * *} p<0.001$. BMI, body mass index; WHR, waist-hip ratio; BIA, bioelectrical impedance analysis; atherosclerosis score, semi-quantitatively assessed rate of atherosclerotic involvement of visceral arteries imaged by MRI; Baecke score, Baecke score of habitual physical activity; $\mathrm{SI}$, insulin sensitivity; $\mathrm{AIRg}$, acute insulin response to glucose; $\mathrm{DI}$, disposition index as per FSIVGTT; HbA1c, glycated hemoglobin; HOMA-IR, homeostatic model assessment for insulin resistance.

To eliminate differences in the deposition of the contrast agent into the bloodstream, a time of 0 was set at the moment of maximum signal intensity in the aorta in order to assess pancreatic perfusion. Then, the area under the curve (AUC), the time required to reach the maximum signal strength, the value of the maximum signal intensity, and the direction of the regression line intersected by the ascending phase of the curve were evaluated for each curve.

In contrast scans, the same experienced radiologist semi-quantitatively assessed the degree of atherosclerotic involvement of the captured part of the abdominal aorta, superior mesenteric artery, and renal arteries. The changes were scored as follows: (1) no signs of atherosclerotic changes, (2) isolated mild lumen irregularities, and (3) significant stenosis of visceral arteries or large plates on the aorta.

\section{Statistical Analysis}

Statistical data processing was performed using GraphPad Prism software (version 6.00 for Windows; GraphPad Software, La Jolla, CA, USA). Unpaired Student's $t$ test, linear regression, and two-way analysis of variance of data were calculated. A significance level of 0.05 was used for all tests.

\section{Results}

All participants underwent full protocol without any adverse events. Groups were comparable in terms of BMI, WHR, and amount of VAT. Both groups also had similar SI, but not fasting glucose and insulinemia levels and cal- culated HOMA-IR. Groups were clearly different in terms of insulin secretion measures, that is, $\mathrm{AIR}_{\mathrm{G}}$ and $\mathrm{DI}$ (shown in Table 1). No subject showed any severe atherosclerotic changes in the visceral arteries on MR scans (score 3 in our scale). Slight inequalities (score 2 ) occurred with equal frequency in both groups $(p=0.726)$. Baecke score of habitual physical activity did not differ between groups, and it did not statistically significantly correlated with pancreatic perfusion values ( $\mathrm{AUC}_{\mathrm{TSIC} \text { mean }}$ ) $(r=0.067, p=0.724)$.

\section{Accumulation of VAT but Not Diabetes Is Associated} with Decline in Pancreatic Perfusion

Statistically significant correlation was found when comparing the AUC time-signal intensity curves of all parts of the pancreas $\left(\mathrm{AUC}_{\mathrm{TSIC} \text { mean }}\right)$ with BMI $(r=-0.434$, $p=0.017)$, WHR $(r=-0.411, p=0.024)$, and areas of VAT calculated from transverse sections of magnetic resonance at the level of the lumbar vertebrae L2-L5 ( $r=$ $-0.436, p=0.016$ ) (shown in Fig. 1) in the whole sample regardless of the diagnosis of T2DM. Moreover, a trend toward significance was found in $\mathrm{AUC}_{\mathrm{TSIC} \text { mean }}$ relation to HOMA-IR $(r=-0.353, p=0.056 \mathrm{NS})$. AUC $\mathrm{ASIC}_{\text {mean }}$ positively correlated with HDL level $(r=0.377, p=0.044)$, but was not statistically significantly related to glycemia $(r=-0.336, p=0.070), \mathrm{HbA1c}(r=-0.312, p=0.093)$, 


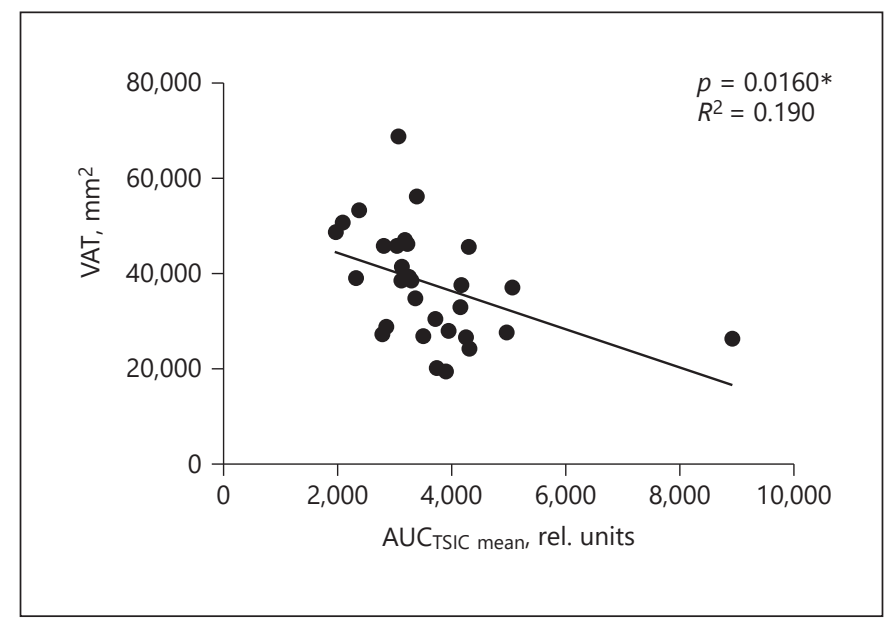

Fig. 1. Perfusion of the pancreas versus visceral adipose tissue. Linear regression was used to test significance, ${ }^{*} p<0.05$. VAT, visceral adipose tissue; $\mathrm{AUC}_{\mathrm{TSIC} \text { mean }}$, area under curve of time/signal intensity curves constructed from DCE-MRI examination; DCEMRI, dynamic contrast-enhanced magnetic resonance imaging.

HDL/cholesterol ratio $(r=0.268, p=0.152)$, age $(r=$ $-0.191, p=0.313)$, or systolic blood pressure $(r=0.087, p$ $=0.647$ ).

Individuals with higher BMI $(r=-0.366, p=0.047)$, WHR $(r=-0.378, p=0.039)$, and volume of VAT in the L2-L5 region $(r=-0.388, p=0.034)$ achieved lower maximum flow rates in the whole pancreas. And further individuals who had a larger area of visceral fat had significantly worse FSIVGTT-derived insulin sensitivity (SI) $(r$ $=-0.531, p=0.003)$ and a lower DI $(r=-0.375, p=0.041)$, and calculated HOMA-IR $(r=0.535, p=0.002)$.

Perfusion Parameters in the Head of the Pancreas Are Related to Fasting Blood Glucose and HOMA-IR

In both of our groups, $\mathrm{AUC}_{\mathrm{TSIC}}$ in the head of the pancreas was negatively correlated with the level of fasting glycemia $(r=-0.401, p=0.028)$. AUC $_{\text {TSIC }}$ in the head of the pancreas correlates also with HOMA-IR $(r=-0.376$, $p=0.041)$, with level of triglycerides $(r=-0.425, p=$ $0.019)$ negatively and HDL $(r=0.400, p=0.029)$ positively in both groups. These correlations were not found in the body and tail of the pancreas. No association was observed either between the parameters of insulin secretion determined by FSIVGTT and the parameters of pancreatic perfusion.

No statistically significant difference was found between the groups in any of the evaluated parameters of pancreatic perfusion (time required to reach the maxi-

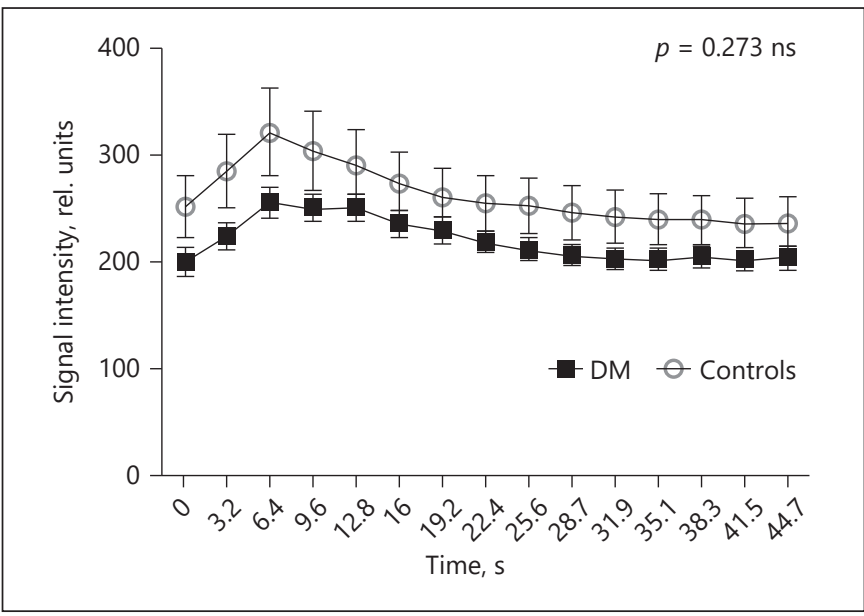

Fig. 2. Time/signal intensity curves in pancreas constructed from DCE-MRI examination; two-way ANOVA was used to test significance, ns $p>0.05$. DCE-MRI, dynamic contrast-enhanced magnetic resonance imaging.

mum signal strength, value of the maximum signal intensity, and direction of the regression line intersected by the ascending phase of the curve). There was also no statistically significant difference when comparing the AUCs using analysis of variance (shown in Fig. 2).

\section{Discussion}

Major finding of the current study is that pancreatic blood flow does relate neither to the presence of diabetes nor it is associated with acute insulin response. We further demonstrated that individuals with higher accumulation of VAT have impaired pancreatic perfusion. Individuals with poorer insulin sensitivity also showed worse perfusion in the head of pancreas. However, we did not find any difference in pancreatic perfusion between obese subjects with diabetes and obese subjects without impaired glucose metabolism, although insulin secretion was unsurprisingly deteriorated in diabetic patients. Also, individuals with higher accumulation of VAT had impaired insulin sensitivity.

Consistently with our findings, Honka in his PET-CT study using radiowater $\left(\left[{ }^{15} \mathrm{O}\right] \mathrm{H}_{2} \mathrm{O}\right)$ and MRI showed a reduction in pancreatic blood flow in morbidly obese patients when compared to lean counterparts depending on the amount of intra-abdominal fat and the fat content in the pancreas. This impaired blood flow was also associ- 
ated with impaired beta-cell function defined as OGTT $\Delta \mathrm{Ins}_{0-30} / \Delta \mathrm{gluc}_{0-30}$ [13]. Later, contrary to expectations, the authors found a further reduction in pancreatic perfusion in the same patients 6 months after bariatric surgery. They explain this by improving insulin sensitivity leading to downregulation of perfusion [14].

Contrary to beta-cell function, we found that some insulin sensitivity measures were related to pancreatic blood flow. HOMA-IR and fasting glycemia both related significantly to blood flow in the head of pancreas, whereas SI did not. This could be explained by the fact that HOMA-IR is based on fasting plasma glucose and insulin levels and reflects therefore hepatic/visceral insulin sensitivity as opposed to SI that assess dynamic glucose disposal of mainly skeletal muscle to glucose load. As VAT has been repeatedly shown to be associated with hepatic insulin resistance [15], we therefore believe that VAT may be a common cause of both insulin resistance and decline in pancreatic blood flow.

\section{Strengths and Limitations}

We present data obtained in well-matched groups, and all our patients with T2DM did not use any medication known to interfere with insulin secretion. For the assessment of glucose metabolism parameters, we used FSIVGTT as a sophisticated and well-proven method.

Quantification of pancreatic perfusion is not easy, and no uniform methodology has been established for it. In our study, we therefore relied on radiological methods, which have already been used experimentally in human medicine and thanks to which it was possible to observe changes in pancreatic perfusion in acute and chronic pancreatitis $[7,16]$.

Atherosclerosis in visceral arteries may be a major confounder when it comes to perfusion. We excluded volunteers having medical history and/or medical report of any atherosclerotic or cardiovascular complication. Moreover, we examined volunteers using pulse wave velocity, which has been validated for arterial stiffness assessment but was shown recently to correlate well with CVD risk [17]. Stenosis and large atherosclerotic plaques on visceral arteries were also ruled out in all subjects on contrast MR scans. However, we acknowledge that the limitation of this method is the impossibility of detecting calcifications using MR.

Because the groups in our study were small and the variability of the perfusion measurements was high, our results comparing the diabetic group and the healthy obese group were evaluated as underpowered by post hoc power analysis (power 0.3 to detect). Also in the group of healthy obese, one subject had a very high pancreatic perfusion compared to the others (shown in Fig. 1). However, even after the elimination of this entity, our presented results remain significant.

Based on our research, we concluded that visceral adiposity predicts impaired pancreatic perfusion and decreased insulin sensitivity; the diagnosis of T2DM alone does not appear to be a predictor. However, we believe that long-term impairment of pancreatic perfusion and insulin resistance in obese individuals may have a negative effect on beta-cell viability and function. Further research in this field is undoubtedly needed.

\section{Statement of Ethics}

The research protocols were approved by the Ethical Committee of the University Hospital Kralovske Vinohrady (Approval Number EK-VP/30/0/2016) in accordance with the Declaration of Helsinki, and the study was conducted in accordance with ICHGCP.

\section{Conflict of Interest Statement}

The authors have no conflicts of interest to declare.

\section{Funding Sources}

The study was supported by Charles University, project GA UK No. 698316 and research program PROGRES Q36. The study was also supported by the Grant No. SGS20/167/OHK3/3T/13 of the Czech Technical University in Prague. J.G. is supported by EFSD mentorship grant by AstraZeneca.

\section{Author Contributions}

R.S., A.P., and J.P. carried out the examinations on human subjects, coordinations of the study, and data analyses. J.W. performed MR examination and analysis. J.H. provided technical support and operation of devices. J.G., J.P., and M.A. designed the study and helped to prepare the manuscript. R.S. was responsible for the design of the study, performed statistical analyses, and drafted the manuscript. All authors read and approved the final manuscript.

\section{Data Availability Statement}

Raw data that support the findings of this study are available from the corresponding author upon request. 


\section{References}

1 Anděl $M$, Němcová $V$, Pavlíková $N$, Urbanová $\mathrm{J}$, Cecháková $\mathrm{M}$, Havlová $\mathrm{A}$, et al. [Factors causing damage and destruction of beta-cells of the islets of Langerhans in the pancreas]. Vnitr Lek. 2014 Sep;60(9):684-90.

2 Henderson JR, Moss MC. A morphometric study of the endocrine and exocrine capillaries of the pancreas. Q J Exp Physiol. 1985 Jul; 70(3):347-56.

3 Polak J, Shimoda LA, Drager LF, Undem C, McHugh H, Polotsky VY, et al. Intermittent hypoxia impairs glucose homeostasis in C57BL6/J mice: partial improvement with cessation of the exposure. Sleep. 2013 Oct; 36(10):1483-90B.

4 Iwase M, Uchizono Y, Tashiro K, Goto D, Iida M. Islet hyperperfusion during prediabetic phase in OLETF rats, a model of type 2 diabetes. Diabetes. 2002 Aug;51(8):2530-5.

5 Bloch K, Vennäng J, Lazard D, Vardi P. Different susceptibility of rat pancreatic alpha and beta cells to hypoxia. Histochem Cell Biol. 2012 Jun;137(6):801-10.

6 Cuenod CA, Balvay D. Perfusion and vascular permeability: basic concepts and measurement in DCE-CT and DCE-MRI. Diagn Interv Imaging. 2013 Dec;94(12):1187-204.
7 Bali MA, Metens T, Denolin V, De Maertelaer V, Devière J, Matos C. Pancreatic perfusion: noninvasive quantitative assessment with dynamic contrast-enhanced MR imaging without and with secretin stimulation in healthy volunteers - initial results. Radiology. 2008 Apr;247(1):115-21.

8 Kim JH, Lee JM, Park JH, Kim SC, Joo I, Han $\mathrm{JK}$, et al. Solid pancreatic lesions: characterization by using timing bolus dynamic contrast-enhanced MR imaging assessment - a preliminary study. Radiology. 2013 Jan; 266(1):185-96.

9 Bergman RN. Toward physiological understanding of glucose tolerance: minimal-model approach. Diabetes. 1989 Dec;38(12):151227.

10 Matthews DR, Hosker JP, Rudenski AS, Naylor BA, Treacher DF, Turner RC. Homeostasis model assessment: insulin resistance and $\beta$-cell function from fasting plasma glucose and insulin concentrations in man. Diabetologia. 1985 Jul;28(7):412-9.

11 Baecke JA, Burema J, Frijters JE. A short questionnaire for the measurement of habitual physical activity in epidemiological studies. Am J Clin Nutr. 1982 Nov;36(5):936-42.
12 Steil GM, Volund A, Kahn SE, Bergman RN. Reduced sample number for calculation of insulin sensitivity and glucose effectiveness from the minimal model. Suitability for use in population studies. Diabetes. 1993 Feb;42(2): 250-6.

13 Honka H, Hannukainen JC, Tarkia M, Karlsson H, Saunavaara V, Salminen P, et al. Pancreatic metabolism, blood flow, and $\beta$-cell function in obese humans. J Clin Endocrinol Metab. 2014 Jun;99(6):E981-90.

14 Honka H, Koffert J, Hannukainen JC, Tuulari JJ, Karlsson HK, Immonen H, et al. The effects of bariatric surgery on pancreatic lipid metabolism and blood flow. J Clin Endocrinol Metab. 2015 May;100(5):2015-23.

15 Gastaldelli A, Cusi K, Pettiti M, Hardies J, Miyazaki $Y$, Berria R, et al. Relationship between hepatic/visceral fat and hepatic insulin resistance in nondiabetic and type 2 diabetic subjects. Gastroenterology. 2007 Aug; 133(2): 496-506

16 Coenegrachts K, Van Steenbergen W, De Keyzer F, Vanbeckevoort D, Bielen D, Chen F, et al. Dynamic contrast-enhanced MRI of the pancreas: initial results in healthy volunteers and patients with chronic pancreatitis. J Magn Reson Imaging. 2004 Dec;20(6):990-7.

17 Kim HL, Kim SH. Pulse wave velocity in atherosclerosis. Front Cardiovasc Med. 2019;6: 41. 